\title{
A new curriculum based on four pillars
}

\author{
N. Ruysserss
}

\section{Introduction}

A few months ago, I was asked by our faculty administration to write a paper about medical education at the University of Antwerp in Flanders, the Dutch speaking part of Belgium. They thought I was the right person for this assignment because not only do I know the faculty of medicine as a student, I also participated in the development of the education programme. In the following, I will highlight some topics which I think are unique to medical education in Flanders.

\section{The new curriculum and the bachelor-master structure}

Over the past three years, professors of our faculty, together with a group of students, have developed a renewed and improved curriculum, aimed at educating better basic doctors who have the competencies required to enter further training, either in one of the clinical specialities, in family medicine, social medicine or in research. In 2006 the new curriculum was first implemented in the first year of the bachelor programme and over the following years it was gradually extended until today only the students in year 5 (master 2) are still following the old curriculum.

The new curriculum is built on four pillars. The first one is the knowledge pillar, consisting of basic science courses and modules. The second pillar is the scientific pillar, which introduces students to research, laboratory work, writing scientific English, data analysis, etc. Then there is the doctor \& society pillar, which focuses on the role of the doctor in contemporary society, and the fourth, clinical pillar deals with clinical and communication skills.

The clinical pillar is the showpiece of medical education at Antwerp. It is aimed at equipping students with clinical skills and preparing them for their future practice as medical professionals. Additionally, the clinical pillar includes learning how to communicate with patients in hospital and primary care settings. A specially trained communication team organizes these courses, comprising theoretical sessions and sessions with simulated patients. In the latter sessions, students practise the skills learned during the theory sessions, such as breaking bad news to patients. Afterwards students receive feedback from the teachers, the simulated patients and their peers. Clinical skills, such as abdominal examination, CPR, suturing, catheterization, paediatric examination, etc. are taught by the 'practice team.'

The implementation of the bachelor master structure was used to create two phases in the seven-year undergraduate medical programme: the bachelor phase and the master phase. The programme of the three-year bachelor phase covers all aspects of the healthy human being but includes also some important clinical syndromes. The second phase comprises the four master years. In master years one and two, the main subjects are clinical syndromes, diagnosis and treatment strategies. The sixth year (master 3 ) is a period of clinical training during which students are judged on their clinical skills. The fourth and final master year consists 
of four months of clinical training, courses to refresh basic medical knowledge and a research thesis, which students have to present to a jury.

In this way students gradually learn about human nature, organ systems, health versus disease, clinical syndromes and how to deal with patients and their diseases. They learn how to function as medical practitioners, as colleagues, as communicators, as managers and as healthcare providers.

During the medical programme, students are offered regular experiences in clinical settings and with patients, such as the ten-day nursing internship in one of the hospitals in Antwerp, which is mandatory for all first-year bachelor students. Students are prepared for the internship by training sessions in which they learn the required nursing skills. Bachelor students also visit a rehabilitation centre as part of the module 'addictions' and master students visit a care home and a nursing home as well as a relief centre for people with dementia. In addition master students attend so-called viewing days in a hospital. During these days they observe consultations and surgical procedures but they are not allowed to actually participate. All these activities plus the clinical training sessions enable students to find out whether being a doctor really is their chosen future profession.

\section{A project worth mentioning}

The practice team that was mentioned earlier also organizes extracurricular weekly 'return days,' on which students can refresh and retrain clinical skills learned in earlier phases of the programme, such as CPR, paediatric examination, etc. The underlying idea of these return days is: the more opportunities students have to practise their skills, the better they will become at performing them.
Another part of this special project is that master students can volunteer for a function as a 'studoc'. After receiving individual training from clinical faculty, 'studocs' are able to work as semi-professionals in the practice team on return days. They are called semi-professionals because, although they are students, they know more than their peers about the skills for which they have received special training.

\section{Where education meets culture}

A project that is currently receiving a lot of attention is internationalization of medical education. Last year English language modules were first introduced for master students. The purpose of these modules is to attract students from all over the world to our Flemish universities. Even though this project is still in its infancy, there is considerable enthusiasm among our professors and students to develop it further in order to make Flemish medical education attractive for all interested students. Another aim of the English language modules is to encourage Belgian students to go abroad to undertake international internships and to study at other universities.

\section{Medicine is more than just studying}

Students at Antwerp are taught in an open and friendly educational climate. Efforts are made to promote good relationships between students and teachers in order to avoid the kind of situation where students are afraid to approach their teachers after class to ask questions or to make an appointment to have certain topics clarified which they did not understand fully in class. In Antwerp the educational climate is characterized by cooperation between students and teachers rather than a hierarchical structure. We already mentioned one factor that 
contributes to this sense of cooperation, namely the possibility for students to take part in the development and organization of the medical education programme. Students can volunteer to join one of the many faculty boards and participate in meetings which are generally scheduled after education sessions. These extracurricular activities give students the opportunity to develop other than medical skills, such as skills for decision making, teamwork and reaching compromises.

And last but not least, our students are offered recreation and relaxation facilities as a 'reward' for their hard work. On Wednesday afternoons no educational sessions are scheduled to enable students to engage in sports or just relax in any way they like. Our student association, 'Aesculapia,' plays a prominent role in this by organizing parties, sports activities, cantus, annual skiing holidays, etc. They ensure wonderful and unforgettable moments, thereby contributing to an enjoyable and memorable university experience.

\section{Conclusion}

Speaking from my experience as a student at Antwerp medical university, I can say that medical education here offers a good balance between studying hard and enjoying student life. The education and training programme offers medical students excellent theoretical and practical preparation for their future professional lives as doctors. In addition, students also learn how to organize their schedules, work together with colleagues and take time to relax. It is this combination that makes studying medicine at Antwerp such a rewarding and satisfying experience.

The author

Natacha Ruyssers, student in medicine, master year 6, former president of SOGA (student board of the faculty of medicine Antwerp), Belgium.

Correspondence

Natacha.ruyssers@student.ua.ac.be

No potential conflict of interest relevant to this article was reported 